\title{
Ki67 Frequency in Breast Cancers without Axillary Lymph Node Involvement and its Relation with Disease-free Survival
}

\author{
Fatemeh Homaei Shandiz ${ }^{1 \&}$, Hossein Shabahang ${ }^{2 \&}$, Fahimeh Afzaljavan ${ }^{3,4 \&}$, \\ Nourieh Sharifi ${ }^{5}$,Alireza Tavasoli ${ }^{6}$, Monavar Afzalaghaee $^{7}$, Emane Roshanzamir $^{8}$, \\ Alireza Pasdar ${ }^{3,9 *}$
}

\begin{abstract}
Background: Breast cancer prognosis is influenced by several histopathology and clinical factors including expression of Ki67 which may have a predictive role in lymph node negative breast cancer patients. The aim of this study was to assess Ki67 expression in breast cancers without axillary lymph node involvement and to evaluate its prognostic value with regard to disease-free survival. Materials and Methods: Subjects were selected from non-metastatic invasive breast cancer patients who were referred to the oncology department of Ghaem hospital during 1 April 2001 to 1 April 2008. Ki67 levels were measured using immunohistochemistry (IHC) and compared with clinicopathological features. The relation of Ki67 expression with disease-free survival was also analysed. Results: A total of 106 women with a mean age of 49 were examined. Some $94.3 \%$ were classified as having invasive ductal carcinomas and the mean tumour diameter at the time of diagnosis was $2.8 \mathrm{~cm}$. Some $50.9 \%$ of cases were ER positive and $47.2 \%$ were PR positive. P53 expression was positive in $48.1 \%$ of the cases. According to the IHC results, only $8.5 \%$ of the patients were Her2/neu positive. Ki67 was positive in $66(62.3 \%)$ with a significant relation to lower age $(p=0.0229)$ and $P 53$ positivity $(p=0.005)$. After an average of 40 -months follow up, $13(12.3 \%)$ demonstrated recurrence, most commonly systemic. Of 13 cases with relapse, 10 patients (77\%) were Ki67 positive. Conclusions: In our population Ki67 appeared to be an independent prognostic factor for three-year survival. However, we stress that a survival study with a bigger sample size would help to support this conclusion.
\end{abstract}

Keywords: Breast cancer - Ki67 - negative axillary lymph node - disease-free survival

Asian Pac J Cancer Prev, 17 (3), 1347-1350

\section{Introduction}

Breast cancer is the most common cancer in women globally and the second cause of cancer-related death in Iranian women (Mousavi et al., 2009). Although there has been no routine screening program in Iran until recently, some breast cancer cases can be diagnosed in the early stage of breast cancer. Therefore, it is essential to evaluate prognostic and especially predictive factors to choose the optimal adjuvant therapy for an individual patient based on established predictive factors.

The prognosis of breast cancer is influenced by several histopathology and clinical factors (Tanriverdi et al., 2014). Axiliary lymph node involvement is one of the most important factors for prognosis and selection of the treatment method (Wexler, 2003). In patients without lymph node involvement, several factors such as tumour size, histopathology grade, patient's age, oestrogen receptor, progesterone receptor, human epidermal growth factor 2 (HER2) and invasion to lymph and blood vessels have prognostic and predictive values for treatment (Mirza et al., 2002). Ki67 as an immunologic proliferation marker, has probably a predictive effect in lymph node negative breast cancer patients (Rossi et al., 2015).

There are several genetic tests to help with selecting treatment method such as Oncotype diagnostic test (21genes tests) which is commercially available (Kittaneh et al., 2013). Unfortunately these tests are expensive and

${ }^{1}$ Department of Radiotherapy-Oncology, Omid and Ghaem Hospitals, ${ }^{2}$ Endoscopic \& Minimally Invasive Surgery Research Center, Ghaem Hospital, ${ }^{3}$ Department of Modern Sciences \& Technologies, Faculty of Medicine, ${ }^{4}$ Student Research Committee, Faculty of Medicine, ${ }^{5}$ Department of Pathology, Faculty of Medicine, ${ }^{6}$ Endoscopic and Minimally Invasive Research Center, Qaem Hospital, ${ }^{7}$ Department of Social Medicine, ${ }^{8}$ Department of Radiotherapy-Oncology, Ghaem Hospitals, Mashhad University of Medical Sciences, Mashhad, Iran, ${ }^{9}$ Division of Applied Medicine, Medical School, University of Aberdeen, Aberdeen, UK *For correspondence: Pasdara@mums.ac.ir 
are not validated and also available in some countries . However, assessment of of some of these markers such as nuclear antigen Ki67, as a cell proliferating marker, is possible using immunohistochemistry. In this study, we assessed the value of Ki67 prognostic factor in 3 years of disease-free survival in breast cancer patients and its relation with other prognostic factors such as age, size of tumour, pathologic subclass, ER, PR, P53 and HER-2/ neu status to see if it can be used as a prognostic factor in decision making for the most appropriate treatment.

\section{Materials and Methods}

In a retrospective cohort study, 106 cases of nonmetastatic invasive breast cancer who were refered to the oncology department of Ghaem hospital from 1 April 2001 to 1 April 2008 were selected according to the following criteria: All of them, had no lymph node involvement where at least 6 axillary lymph node dissections were negative. Ki67 assesment was done by the assigned pathologist for all samples using immunohistochemistry (IHC) method. All patients received adjuvant therapy according to the routine guidelines. Clinicopathological data including sex, age, time of the diagnosis and surgery, pathologic subtype, tumour size and staging, ER, PR, P53, HER2/neu status were also collected. All patients were followed up until 1 April 2011; follow up duration, time of surgery and the last referral time to the oncology center were recorded on a monthly time scale. Local recurrence in the same side, regional recurrence in the regional lymph node involving axillary, supra and infra clavicular and systemic recurrence as distant metastasis to the other organs were defined. If there were any recurrence during the follow up period, the interval between surgery and recurrence would be calculated in month as disease-free survival.

According to the previous studies, ER and PR more than 1\% (2010), p53 more than 10\% (Stal et al., 1995), HER2/neu 3+ (Wolff et al., 2014) and Ki67 more than $10 \%$ by IHC were considered positive (Moriya et al., 2000). With respect to the fact that there is no agreement about certain Ki67 proliferative factor levels, the results were examined separately using two different cut-off points; Ki67>10\% and $\mathrm{Ki} 67>20 \%$, however, in this study Ki67 >20\% was considered as positive. Tumour staging ( $\mathrm{T})$ was performed using the available tumour size in the pathology report.

\section{Statistical Analysis}

All data were analyzed using SPSS software (version 13). Kaplan-Meier survival analysis was used to compare survival in two groups, Ki67 positive and negative. Chisquare was used to compare the others prognostic factors and Ki67 status. A p-value less than 0.05 was considered statistically significant.

\section{Results}

In this study, 106 non-metastatic invasive breast cancer patients without lymph node involvement were assessed. They were aged between 28 and 79 years with the mean age $49 \pm 5$ and were followed up between 4 to 109 months (the mean follow up duration was 40 months). The clinicopathological features are shown in Table 1.

$94.3 \%$ of patients were invasive ductal carcinoma and the mean size of the tumour at the time of diagnosis was 2.8 centimeter. $50.9 \%$ of patients were ER positive and $47.2 \%$ of them were PR positive and P53 was positive in $48.1 \%$ of the cases. According to the IHC results, only $8.5 \%$ of patients were Her2/neu positive.

The assessment results of Ki67 by IHC method according to the two different cut-off values are shown in Table 2.

In this study, $\mathrm{Ki} 67 \geq 20 \%$ was considered as positive, so $62.3 \%$ of patients were Ki67 positive. The mean age of the patients was $53 \pm 11$ for negative Ki67 and $47 \pm 11$ for positive ones. Results indicated a significant relation between Ki67 and age ( $p=0.0229)$. In both conditions the

Table 1. Clinicopathological Features in Breast Cancer Patients

\begin{tabular}{|c|c|c|}
\hline $\begin{array}{c}\text { clinicopathological } \\
\text { features }\end{array}$ & Type & $\begin{array}{c}\text { Number } \\
\text { (percentage) }\end{array}$ \\
\hline \multirow{3}{*}{ Tumour variant } & Ductal & $100(94.3)$ \\
\hline & Lobular & $6(5.7)$ \\
\hline & Total & $106(100)$ \\
\hline \multirow{3}{*}{ Tumour location } & Right breast & $45(42.5)$ \\
\hline & Left breast & $61(57.5)$ \\
\hline & Total & $106(100)$ \\
\hline \multirow{2}{*}{ Grade } & Grade1 & $12(10.9)$ \\
\hline & Grade2\&3 & $84(79.1)$ \\
\hline \multirow{3}{*}{ Staging $(\mathrm{T})$} & $\mathrm{T} 1 \leq 2$ & $29(36.8)$ \\
\hline & $\mathrm{T} 2(2-5)$ & $67(63.2)$ \\
\hline & Total & $106(100)$ \\
\hline \multirow{3}{*}{ Oestrogen receptor } & Negative & $52(49.1)$ \\
\hline & Positive & $54(50.9)$ \\
\hline & Total & $106(100)$ \\
\hline \multirow{3}{*}{$\begin{array}{l}\text { Progesterone recep- } \\
\text { tor }\end{array}$} & Negative & $56(52.8)$ \\
\hline & Positive & $50(47.2)$ \\
\hline & Total & $106(100)$ \\
\hline \multirow{3}{*}{ p53 } & Negative & $55(51.9)$ \\
\hline & Positive & $51(48.1)$ \\
\hline & Total & $106(100)$ \\
\hline \multirow{3}{*}{ HER2/neu } & Negative & $97(91.5)$ \\
\hline & Positive & $9(8.5)$ \\
\hline & Total & $106(100)$ \\
\hline
\end{tabular}

Table 2. Frequency of Ki67 Positive Patients Based on Threshould

\begin{tabular}{lccc}
\hline Ki67 staus & $\begin{array}{c}\text { Negative } \\
\text { No. }(\%)\end{array}$ & $\begin{array}{c}\text { Positive } \\
\text { No. }(\%)\end{array}$ & $\begin{array}{c}\text { Total } \\
\text { No. }(\%)\end{array}$ \\
\hline Ki67 $\geq 20 \%:$ & $40(37.7)$ & $66(62.3)$ & $106(100)$ \\
Ki67 $\geq 10 \%:$ & $22(20.8)$ & $84(79.2)$ & $106(100)$ \\
\hline
\end{tabular}

Table 3. Frequency Distribution of Recurrence Region in Patients

\begin{tabular}{lc}
\hline Recurrence region: & Number (Percentage) \\
\hline Local & $4(30.75)$ \\
Regional & $0(0.0)$ \\
Systemic & $7(53.84)$ \\
Local and regional & $1(7.7)$ \\
Local and systemic & $1(7.7)$ \\
Total & $13(100)$ \\
\hline
\end{tabular}




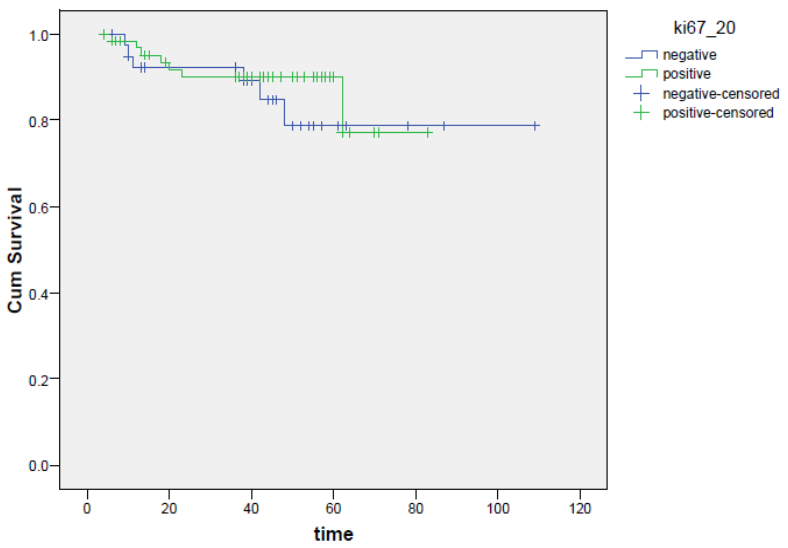

Figure 1. Disease-free Survival According to Ki67 Results in Patients

mean age of Ki67 positive ones was less than that of the Ki67 negative ones.

There was no significant difference between the tumour size in negative and positive Ki67 cases ( $\mathrm{p}=0.237)$.

After an average of 40 month follow up, 13 patients $(12.3 \%)$ had recurrence of which 10 patients $(77 \%)$ were Ki67 positive ( $p=0.0235)$. The region of recurrences is shown in Table 3 . The most common kind of recurrence was distant metastasis in the cases with recurrence.

According to the Kaplan-Meier test in patients with Ki67 $\leq 20 \%$, the 3-year disease-free survival period was $89 \%$ and in patients with Ki67>20\%, it was $85 \%$ (Figure $1)$.

Mean survival period in patients with $\mathrm{Ki} 67 \leq 20 \%$ was $92 \pm 6$ months and 73/5 \pm 3 months for Ki67>20\%. Logistic regression analysis showed no significant difference between the two groups ( $\mathrm{p}=0.556)$.

Moreover, Chi-square was used to compare the other prognostic factors with Ki67 status. With respect to the fact that age in both groups with Ki67 positive and negative had a significant difference, we entered this factor accompanied with Ki67>20\%, into the Cox regression, and the result did not show any relation with the age effect, nor with the survival period.

\section{Discussion}

In this study, the value of Ki67 prognostic factor in a 3-year of disease-free survival in breast cancer patients and its relation with other prognostic factors were assessed.

With regard to the fact that there are no agreements about certain Ki67 proliferative factor levels, in the current study, we considered $\mathrm{Ki} 67>20 \%$ as positive according to the proposed scale in the study done by Colleoni, $\mathrm{M}$. et al. (Colleoni et al., 2004).

The mean age of Ki67 negative patients was $49 \pm 11$. We found a significant relation between $\mathrm{Ki67}$ and age ( $\mathrm{p}=0.0229)$ where the mean age of Ki67 positive patients was less than that of Ki67 negatives'. Previously, it has been shown there was a strong relation between Ki67 and age (Sahin et al., 1991).

The mean tumour sizes in Ki67 negative and positive cases were $2.79 \mathrm{~cm}$ and $2.88 \mathrm{~cm}$ respectively, so the mean tumour size in Ki67 positive cases was more than that of the negative ones. However, there was no significant relation between Ki67 and tumour size ( $\mathrm{p}=0.581)$. Previous studies indicated contravertial results on this issue. The results of some studies showed that Ki67 had a strong relation with tumour size (Gonzalez-Sistal et al., 2014). Furthermore, the results of other studies were different in which there were no relations between Ki67 and tumour size (Haroon et al., 2013; Knutsvik et al., 2014).

According to our results, there was not any correlation between Ki67 and oestrogen receptor $(\mathrm{P}$ value $=0.029)$, which was similar to Haroon S et al. (Haroon et al., 2013). This is in contrast with two other studies where there was a reverse relation between ER and Ki67 (Li et al., 2014). In addition, no correlation was found between $\mathrm{Ki} 67$ and progesterone receptor in this study (Pvalue $=0.112$ ) while previous studies showed an inverse correlation (Haroon et al., 2013; Wong et al., 2014).

In our study there was a significant relation between $\mathrm{Ki} 67$ and p53 expression ( $\mathrm{P}$ value=0.005). It has been suggested that overexpression of p53 is due to frequent mutations and overexpression is associated with higher Ki67 (Sirvent et al., 1995). In this study, there was no relation between Ki67 and HER2/neu oncogene (Pvalue $=0 / 47$ ) which is similar to Osman I et al. (Osman et al., 2001).

The mean survival rate was $92 \pm 6$ months in Ki67 negative cases and $73 \pm 3$ in positive ones, respectively. However, this difference was not significant $(\mathrm{p}=0.556)$. Considering the fact that age had a significant relation with Ki67 in both groups, survival rate was assessed by removing the age from the equation to omit the age effect. However, some reports indicated that Ki67 did not have any prognostic value, and there was no relation between Ki67 and survival rate (Payandeh et al., 2014). In contrast, as mentioned before, in many other studies, the prognostic value of $\mathrm{Ki} 67$ has been frequently reported and this protein has been introduced as a predictive marker in recurrence (Kontzoglou et al., 2013; Inwald et al., 2013).

In this study, Ki67 was positive in 10 patients with recurrence $(77 \%)$ and there was a significant correlation between recurrence and $\mathrm{Ki} 67$ status $(\mathrm{p}=0.0235)$. In a meta-analysis by Azambuja, it has been suggested that Ki67 is a recurrence marker in patients' survival with primary breast cancer (de Azambuja et al., 2007). In another study after assessing 669 cases during 53 months, researchers proposed that low rate of proliferation in invasive breast cancer without lymph node involvement is a good prognostic sign (Jones et al., 2001). Several studies have shown that Ki67 provides independent prognostic information especially in breast cancer without lymph node involvement (Mirza et al., 2002) and higher Ki67 was associated with a lower disease-free survival (Kilickap et al., 2014). Therefore, in our population Ki67 can be used as an independent prognostic factor.

One of the current limitations which may justify some differences in results of this study with other similar ones is the limited number of the studied patients. This was due to the low number of patients who met the criteria as we considered at least examination of six axillary lymph nodes. In most of the cases the number of the evaluated 
lymph nodes was uncertain or less than six, so they were not assessable for the study. In addition, the follow up period in 18 cases was less than 36 months, therfore it makes the exact judgment about recurrence difficult in these cases as the recuurence might be likely after a longer period.

In conclusion, according to this study, although there is not a significant relation between Ki67 and 3-year disease-free survival, there is a significant relation between Ki67 and recurrence rate and also with some prognostic factors such as P53 status and age. We suggest if it would be available, a physician should use standard tests such as Oncotype for the assessment of the recurrence risk, otherwise prognostic factors such as Ki67 may be helpful.

Further assessment is needed to evaluate the relationship between $\mathrm{Ki} 67$ and the recurrence risk. More precise results can be achieved by analyzing more subjects, paying more attention to the lymph node involvement status and increasing the follow up duration. Performing a meta-analysis in this area is recommended as the new results are emerging.

\section{Acknowledgements}

We would also like to thank oncology dependent staff at Ghaem Hospital, Mashhad University of Medical Sciences for their helpful contribution.

\section{References}

Colleoni M, Rotmensz N, Peruzzotti G, et al (2004). Minimal and small size invasive breast cancer with no axillary lymph node involvement: the need for tailored adjuvant therapies. Ann Oncol, 15, 1633-9.

de Azambuja E, Cardoso F, de Castro G, et al (2007). Ki-67 as prognostic marker in early breast cancer: a meta-analysis of published studies involving 12,155 patients. Br J Cancer, 96, 1504-13.

Gonzalez-Sistal A, Sanchez AB, Del Rio MC, et al (2014). Association between tumor size and immunohistochemical expression of Ki-67,p53 and BCL2 in a node-negative breast cancer population selected from a breast cancer screening program. Anticancer Res, 34, 269-73.

Haroon S, Hashmi AA, Khurshid A, et al (2013). Ki67 index in breast cancer: correlation with other prognostic markers and potential in pakistani patients. Asian Pac J Cancer Prev, 14, 4353-8.

Hezova R, Slaby O, Faltejskova P, et al (2010). microRNA-342, microRNA-191 and microRNA-510 are differentially expressed in $\mathrm{T}$ regulatory cells of type 1 diabetic patients. Cell Immunol, 260, 70-4.

Inwald EC, Klinkhammer-Schalke M, Hofstädter F, et al (2013). $\mathrm{Ki}-67$ is a prognostic parameter in breast cancer patients: results of a large population-based cohort of a cancer registry. Breast Cancer Res Treat, 139, 539-52.

Jones S, Clark G, Koleszar S, et al (2001). Low proliferative rate of invasive node-negative breast cancer predicts for a favorable outcome: a prospective evaluation of 669 patients. Clin Breast Cancer, 1, 310-4.

Kilickap S, Kaya Y, Yucel B, et al (2014). Higher Ki67 expression is associates with unfavorable prognostic factors and shorter survival in breast cancer. Asian Pac J Cancer Prev, 15, 1381-5.

Kittaneh M, Montero AJ, Glück S (2013). Molecular profiling for breast cancer: a comprehensive review. Biomarkers Cancer, 5, 61-70.

Knutsvik G, Stefansson IM, Aziz S, et al (2014). Evaluation of ki67 expression across distinct categories of breast cancer specimens: a population-based study of matched surgical specimens, core needle biopsies and tissue microarrays. PLOS ONE, 9, 112121.

Kontzoglou K, Palla V, Karaolanis G, et al (2013). Correlation between Ki67 and breast cancer prognosis. Oncol, 84, 219-25.

Li FY, Wu SG, Zhou J, et al (2014). Prognostic value of Ki-67 in breast cancer patients with positive axillary lymph nodes: a retrospective cohort study. PLoS One, 9, 87264.

Mirza AN, Mirza NQ, Vlastos G, et al (2002). Prognostic Factors in Node-Negative Breast Cancer: A Review of Studies With Sample Size More Than 200 and Follow-Up More Than 5 Years. Ann Surg, 235, 10-26.

Moriya T, Sakamoto K, Sasano H, et al (2000). Immunohistochemical analysis of Ki-67, p53, p21, and p27 in benign and malignant apocrine lesions of the breast: its correlation to histologic findings in 43 cases. Mod Pathol, 13, 13-8.

Mousavi SM, Gouya MM, Ramazani R, et al (2009). Cancer incidence and mortality in Iran. Ann Oncol, 20, 556-63.

Osman I, Scher HI, Drobnjak M, et al (2001). HER-2/neu (p185neu) protein expression in the natural or treated history of prostate cancer. Clin Cancer Res, 7, 2643-7.

Payandeh M, Sadeghi M, Fekri A, et al (2014). P53 mutation compared with Ki67 marker in metastasis of breast cancer in western. Iran J Solid Tumors, 4, 4-9.

Rossi L, Laas E, Mallon P, et al (2015). Prognostic impact of discrepant Ki67 and mitotic index on hormone receptorpositive, HER2-negative breast carcinoma. Br J Cancer, 113, 996-1002.

Sahin AA, Ro J, Ro JY, et al (1991). Ki-67 immunostaining in node-negative stage I/II breast carcinoma. Significant correlation with prognosis. Cancer, 68, 549-57.

Sirvent JJ, Salvado MT, Santafe M, et al (1995). p53 in breast cancer. Its relation to histological grade, lymph-node status, hormone receptors, cell-proliferation fraction (ki-67) and c-erbB-2. Immunohistochemical study of 153 cases. Histol Histopathol, 10, 531-9.

Stal O, Stenmark Askmalm M, Wingren S, et al (1995). p53 expression and the result of adjuvant therapy of breast cancer. Acta Oncol, 34, 767-70.

Tanriverdi O, Meydan N, Barutca S (2014). Reconsideration of clinical and histopathological prognostic factors in breast cancer patients: a single center experience. Asian Pac J Cancer Prev, 15, 807-12.

Wexler MJ (2003). Role of axillary lymph-node dissection in the management of breast cancer. Canadian J Surg, 46, 247-50.

Wolff AC, Hammond ME, Hicks DG, et al (2014). Recommendations for human epidermal growth factor receptor 2 testing in breast cancer: American Society of Clinical Oncology/College of American Pathologists clinical practice guideline update. Arch Pathol Lab Med, 138, 241-56.

Wong H, Lau S, Cheung P, et al (2014). Lobular breast cancers lack the inverse relationship between ER/PR status and cell growth rate characteristic of ductal cancers in two independent patient cohorts: implications for tumor biology and adjuvant therapy. BMC Cancer, 14, 826 . 